\title{
XRD Doping Control of Light-Emitting cBN with a Large Size Mismatch between the Dopant and Intrinsic Atoms
}

\author{
E. M. Shishonok ${ }^{1}$, V. G. Luhin'1 ${ }^{1}$ J. W. Steeds ${ }^{2}$ \\ ${ }^{1}$ Belarusian State University of Technology, Minsk, Republic of Belarus \\ ${ }^{2}$ School of Physics, University of Bristol, Bristol, UK \\ Email: eshishonok@tut.by
}

How to cite this paper: Shishonok, E.M., Luhin, V.G. and Steeds, J.W. (2016) XRD Doping Control of Light-Emitting cBN with a Large Size Mismatch between the Dopant and Intrinsic Atoms. World Journal of Engineering and Technology, 4, 174-185. http://dx.doi.org/10.4236/wjet.2016.43D021

Received: September 19, 2016

Accepted: October 13, 2016

Published: October 20, 2016

\begin{abstract}
Cubic boron nitride ( $\mathrm{cBN}$ ) as the outstanding representative of the family of semiconducting wide bandgap nitrides and the closest analogue of diamond, is produced and investigated. XRD as method for doping control of $\mathrm{cBN}$ with impurities of large atomic sizes, is suggested. The larger an atomic size mismatch between doping and intrinsic atoms of a semiconductor's crystal lattice, the stronger its response through own strains and distortions. The distortions are expected to be notable in the case of the smallest intrinsic atoms of $\mathrm{cBN}$ and diamond. The light-emitting $\mathrm{cBN}$ doped with various rare-earth elements (RE) in different concentrations under high pressure conditions is synthesized in form of the $\mathrm{cBN}$ : RE single phase micropowders. The micropowders showed the discrete photoluminescence spectra in IR-, red and green spectral ranges which were attributed to the intra-electronic transitions of $\mathrm{RE}^{3+}$ ions located in $\mathrm{cBN}$ crystal lattice. The locations of the $\mathrm{RE}^{3+}$ ions in $\mathrm{cBN}$ crystal lattice are discussed. The data of XRD $\left(\mathrm{Cu}_{\mathrm{Ka}}\right)$ analysis of the $\mathrm{cBN}$ : RE micropowders are represented. Extra-splits (as the additional ones to the $\alpha_{1}-\alpha_{2}$-splits on $\mathrm{Cu}_{\mathrm{Ka}}$ ) of the $\mathrm{cBN}$ parent peaks in XRD patterns of the $\mathrm{CBN}$ : RE, are discovered and analyzed using appropriate computer programs. As established, crystal lattice of $\mathrm{cBN}$ due to the incorporation of $\mathrm{RE}^{3+}$ ions, represents a disordered solid solutions which are nonuniformly distorted in dependence on the ions' size and their concentrations in $\mathrm{cBN}$. Results of the present work can be useful to manufacture $c B N$ with predictable functional properties, as well as for in situ doping control of $\mathrm{cBN}$ and diamond.
\end{abstract}

\section{Keywords}

Cubic Boron Nitride, High Pressure, Rare Earth Element, İon, Concentration, Photoluminescence, XRD Analysis, Crystalline Distortion 


\section{Introduction}

Cubic boron nitride $(\mathrm{cBN})$ is the widest bandgap $\left(\mathrm{E}_{\mathrm{g}}=6.4 \mathrm{eV}\right)$ semiconductor in the AIIIBV group. CBN is the closest analog of diamond but is superior to the latter in the bandgap width, thermal, radiation, and chemical stability, the possibility of forming $n$ and p-type conduction, and the capability of emitting secondary electrons. CBN has been recognized as the unique material promising for the use in opto- and microelectronics, including for the employment in light emitters of various purposes operative in UV, visible, and IR spectral regions. Understandably, the successful production of RE-activated cBN samples exhibiting intense light emission in the IR, visible, and UV spectral ranges, variously stimulated, might open up opportunities for manufacturing new generation light-emitting diodes, solid state lasers, detectors, and phosphors operating under extreme conditions. Rigidity of the crystal lattice of $c B N$ will ensure mechanical strength, radiation stability, and the capability of operating under the conditions of high electric fields for all devices based on $\mathrm{cBN}$. It is expected that the high thermal stability will make $\mathrm{cBN}$ virtually indispensable for the use in passive elements of future electron devices, such as takeoffs of heat from chips with high heat release, by ensuring their high operation speed. A high speed of sound will make it possible to use $\mathrm{CBN}$ in devices operating in a gigacycle range, and the presence of piezoelectric properties will allow its employment in piezo elements, under extreme operational conditions. At last, $\mathrm{cBN}$ has been announced as promising functional material for future quantum computers [1]. Among the main reasons for slow "mastering" of $\mathrm{cBN}$ as a functional material for solid state electronics is the absence of large perfect single crystals of cBN. On the other hand, a good alternative to single crystals of $\mathrm{cBN}$ for opto- and microelectronic applications can be its other morphological forms, like micropowders which, while exhibiting intense light emission (LE) are ready for the utilization by downconversion phosphors.

\section{Experimental Details}

The $c B N: R E$ (RE: rare earth element) yellow micropowders were manufactured under high pressure-high temperature (HPHT) via catalytic synthesis from $\mathrm{hBN}$ in presence of the low-melting compound based on $\mathrm{RE}$ ( $\mathrm{Er}, \mathrm{Tb}, \mathrm{Eu}, \mathrm{Sm}, \mathrm{Nd}$ ). The granulometric analysis of cBN:RE micropowders was provided with "Laser particle size analyzer LS-POP(VI), OMEC INSTRUMENTS CO., LTD”. For all micropowders', the volume mean diameters $\mathrm{D}(4,3) \approx 3.5-5.5 \mu \mathrm{m}$ of grains, were revealed. The grains enlarged in size were observed, when the concentrations of the RE largest ions in $\mathrm{CBN}$ increased. CBN micropowders were analyzed in their phase content using XPS and RBS methods, which data coincided well. If the precursors of cBN contained 0.5 - 15 wt. \% of the RE compound, only from less than 0.01 up to $\sim 0.1$ at. \% of the RE element could be introduced into crystal lattice of $\mathrm{cBN}$ using the concrete catalytic system. XRD patterns of the micropowders were taken repeatedly with recording step of $0.01 \%$ min using D8 Advance Bruker AXS instrument on $\mathrm{Cu}_{\mathrm{Ka}}$ radiation. For measurements, the equal masses of the micropowders were placed into standard cuvettes. The rotation option of 
samples was not used, because of small sizes of the micropowders' grains. Computer programs were used for deconvolution of the XRD patterns' peaks on the minimal number of components, a package of which gave calculated peaks with the $\sim 5 \%$ deviation from the experimental peaks. It was taken into account that the ratio of intensities of $\dot{\alpha}_{1}$ - and $\dot{\alpha}_{2}$-component in $\mathrm{Cu}_{\mathrm{Ka}}$-doublet is equal almost two and the components have the equal half-widths. The contributions of a Lorentzian and a Gaussian into an experimental peak's shape, which both cause either the particle-size broadening or the instrumental and strain-broadening of the peak correspondingly, allowed us to use the Voigt profiles as a natural description of peaks in XRD patterns of the $\mathrm{CBN}$ :RE micropowders.

Generally, photoluminescence (PL) tests of $\mathrm{cBN}$ :RE were performed using RENISHAW-1000 spectrometers at room temperature.

\section{Results and Discussion}

\subsection{Photoluminescence Testing}

The PL discrete spectra $\left(\lambda_{\text {exc }}=488 \mathrm{~nm}\right)$ of $\mathrm{cBN}$ :Eu micropowders with $\sim 0.01, \sim 0.05$ and 0.1 at.\% Eu in $\mathrm{cBN}$ are represented in Figures $1(\mathrm{a})-(\mathrm{c})$. They are originated from the consequence of the radiative electronic transitions ${ }^{5} \mathrm{D}_{0} \rightarrow^{7} \mathrm{D}_{\mathrm{J}}(\mathrm{J}=0,1,2,3,4)$ of $\mathrm{Eu}^{3+}$ ions which are located in cBN crystal lattice in crystalline field of low symmetry [2]. In this work we first can obtain, that headlines of the spectra (the insets in Figures 1(a)-(c)) are visibly split into two separate main lines. It means that $\mathrm{Eu}^{3+}$ ions occupy two sites of

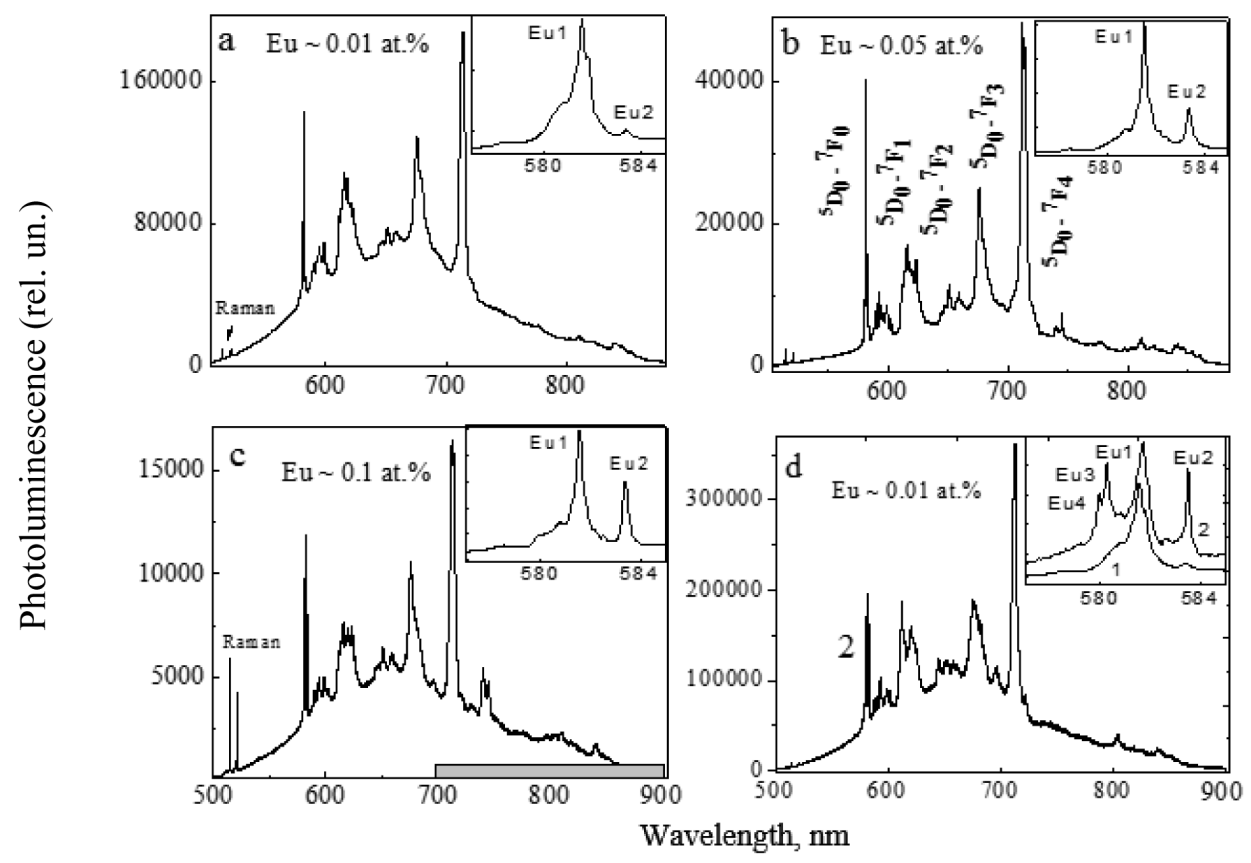

Figure 1. PL spectra of cBN:Eu micropowders with different Eu content in cBN: $\sim 0.01$ (a), $\sim 0.05$ (b) и $\sim 0.1$ at.\% (c), and of cBN:Eu ( 0.01 at.\% Eu) before (1) and after annealing at $\mathrm{T}_{\text {ann }}=870 \mathrm{~K}$ (2) (d) in the inset; $\lambda_{\text {exc }}=488 \mathrm{~nm}, \mathrm{~T}_{\text {reg }}=300 \mathrm{~K}$. 
low symmetry in the cBN crystal lattice which create two luminescence centers (we called them Eu1 and Eu2). It is clear from the insets, that the $\mathrm{Eu}^{3+}$ ions occupy the second site in $\mathrm{cBN}$ crystal lattice the more expectable, the more concentration of Eu in $\mathrm{cBN}$. The two-center character of $\mathrm{Eu}$ in $\mathrm{cBN}$ was suggested in our previous work [3]. The represented data here directly testify to the suggestion. The Eu1 and Eu2 centers have already figured in [3], but they were observed among four luminescence centers in PL spectra of the cBN:Eu ( 0.01 at.\% Eu) micropowder which was annealed at $\mathrm{T}_{\text {ann }}=$ $870 \mathrm{~K}$ (Figure 1(d), the inset). More likely, that the annealing effect is caused by redistribution of $\mathrm{Eu}^{3+}$ ions among sites in $\mathrm{cBN}$ crystal lattice due to activation in it of the nitrogen vacancies' motion [4]. The PL multiband spectra $\left(\lambda_{\text {exc }}=488 \mathrm{~nm}\right)$ of $\mathrm{cBN}: \mathrm{Tb}$ micropowders (Figure 2(a) and the inset) are related here to the ${ }^{5} \mathrm{D}_{4} \rightarrow{ }^{7} \mathrm{~F}_{6,5,4,3}$ intra-electronic transitions of $\mathrm{Tb}^{3+}$ ions. The $\mathrm{cBN}$ : Tb spectrum considerably increases in intensity when the $\mathrm{Tb}$ concentration in $\mathrm{cBN}$ grows from $\sim 0.05$ up to $\sim 0.1$ at. $\%$. In this case, the spectrum remains structurally unchanged. It meant, that $\mathrm{Tb}^{3+}$ ions continue locating in the similar crystallographic positions inside $\mathrm{cBN}$ crystal lattice. The ions stay in the same positions after annealing the $\mathrm{cBN}: \mathrm{Tb}(0.1$ at.\% $\mathrm{Tb})$ at $\mathrm{T}_{\mathrm{ann}}=870 \mathrm{~K}$, in opposite to the annealed cBN:Eu (Figure $1(\mathrm{~d})$ ). However, the PL spectrum of the annealed
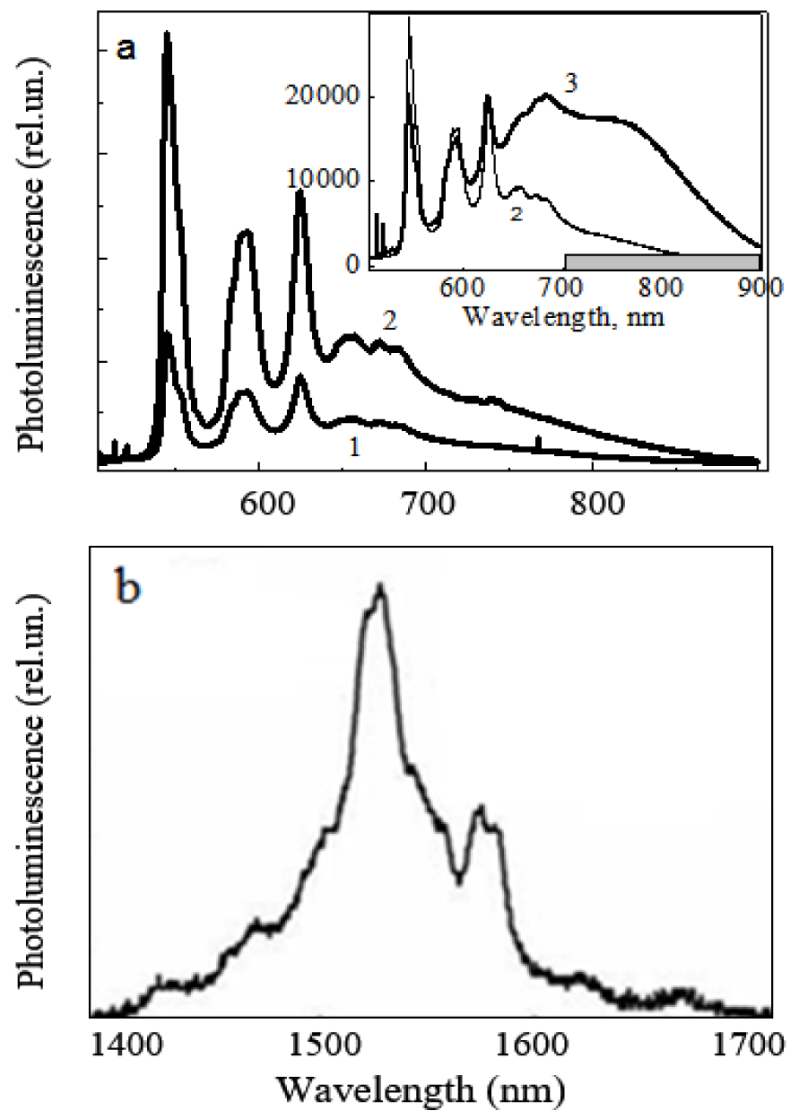

Figure 2. PL spectra of $\mathrm{cBN}: \mathrm{Tb}$ micropowders with different $\mathrm{Tb}$ content $(1-0.05,2-0.1$ at.\% $\mathrm{Tb}$ ) and in the inset-before (2) and after (3) annealing at $\mathrm{T}_{\mathrm{ann}}=870 \mathrm{~K}, \lambda_{\mathrm{exc}}=488 \mathrm{~nm}$ (a); PL spectrum of BN: Er micropowder, $\lambda_{\text {exc }}=960 \mathrm{~nm}(\mathrm{~b}) ; \mathrm{T}_{\mathrm{reg}}=300 \mathrm{~K}$. 
$\mathrm{cBN}: \mathrm{Tb}$ is characterized with appearance of broad band in range of 700-900 nm (the gray strip in Figure 1(d) and in the inset to Figure 2(a)) of the multi-vacancy nature [5]. The PL spectrum in range of $1400-1700 \mathrm{~nm}$ of the $\mathrm{cBN}$ :Er single phase micropowder ( 0.05 at. \% Er) is originated from the ${ }^{4} \mathrm{I}_{13 / 2} \rightarrow{ }^{4} \mathrm{I}_{11 / 2}$ electronic transitions (under the laser diode excitation with $\lambda_{\text {exc }}=960 \mathrm{~nm}$ ) of the $\mathrm{Er}^{3+}$ ions incorporated into $\mathrm{cBN}$ crystal lattice (Figure 2(b)). The PL spectra of the $\mathrm{cBN}$ :Nd micropowders reflects the two-center character of $\mathrm{Nd}^{3+}$ ions in $\mathrm{cBN}$, referring to our work [6]. The $\mathrm{cBN}: \mathrm{Sm}$ micropowders under PL analysis are described by us in [7].

\subsection{XRD Analysis of cBN:RE Micropowders}

XRD patterns of $\mathrm{cBN}$ :RE micropowders showed their single phase nature, because the patterns contained only (111), (200), (220), (311) and (331) peaks which belonged to $\mathrm{cBN}$ with the face-centered cubic lattice (FCC). We will not pay attention here to features of the patterns which were previously discovered by us from the comparative analysis of XRD patterns of $\mathrm{cBN}$ : RE and $\mathrm{cBN}$ standard. The features testify to the formation of disordered solid solutions of rare earth elements ( $\mathrm{Tm}, \mathrm{Tb}, \mathrm{Eu}, \mathrm{Ce})$ on cBN basis [8]. In the present paper via analysis of shapes of peaks in XRD patterns of $\mathrm{cBN}$ :RE, we develop our conceptions about a possible distortions of the $\mathrm{CBN}$ rigid crystal lattice, which can be caused by impurities of large sizes (the $\mathrm{RE}^{3+}$ ions here) of various concentrations. Peaks (111), (220), and (311) in XRD patterns of cBN:RE (in particular of $\mathrm{cBN}: \mathrm{Eu}, \mathrm{cBN}: \mathrm{Nd}$ ) with the RE smallest concentrations and the RE largest concentrations are represented in Figure 3 and Figure 4. Hereinafter, a content in weight percent of any RE compound within a total mass of the precursor, is specified in parentheses next to the content in atomic percent. As seen from Figure 3 and Figure 4 when the concentration of $\mathrm{Eu}$ and $\mathrm{Nd}$ in $\mathrm{cBN}$ increases from less than $\sim 0.01$ at.\% (0.5; 1) up to $\sim 0.05$ at.\% (10), the peaks are decreased in intensity. With the concentration increasing, peaks (111), (220), (311) and (331) are displaced to the low $2 \Theta$ range, testi fying to the disordered solid solutions' creation. We can refer to [8] as well.

As established, all peaks in XRD patterns of all cBN:RE micropowders are variously asymmetric. Mainly, because the low $2 \Theta$-branches of peaks (111), (220) and (311) are enormously stretched towards the low $2 \Theta$-angles, particularly, for cBN with the RE
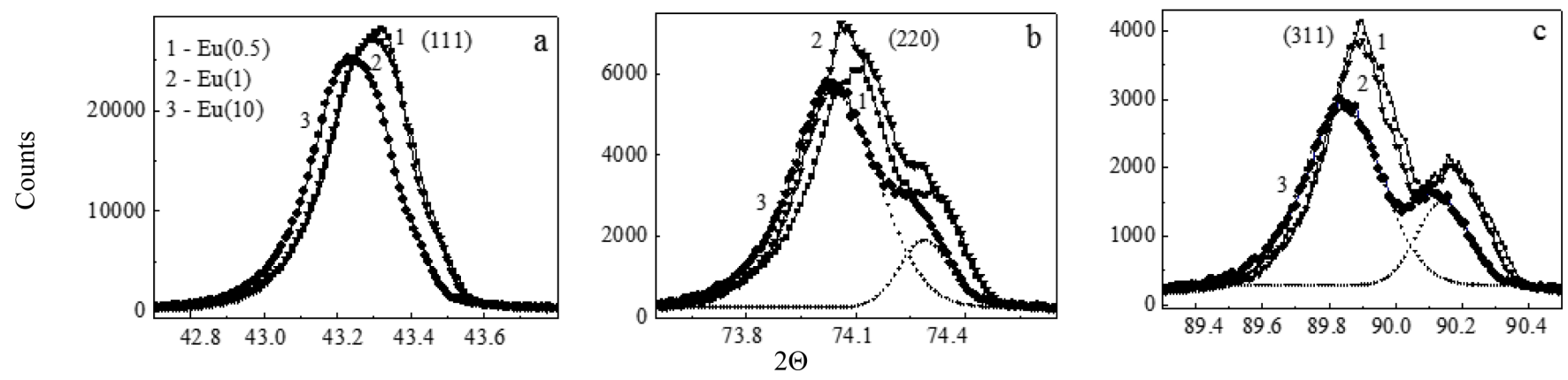

Figure 3. Peaks (111) (a); (220) (b); (311) (c) in XRD patterns of the $\mathrm{cBN}$ :Eu micropowder with the Eu different content in cBN: 1,2 - < 0.01 at.\% (0.5; 1$), 3$ - 0.05 at.\% (10); peaks 3 (b, c) approximated with two Gaussians, which are not $\dot{\alpha}_{1^{-}} \dot{\alpha}_{2^{-}}-$doublets. 

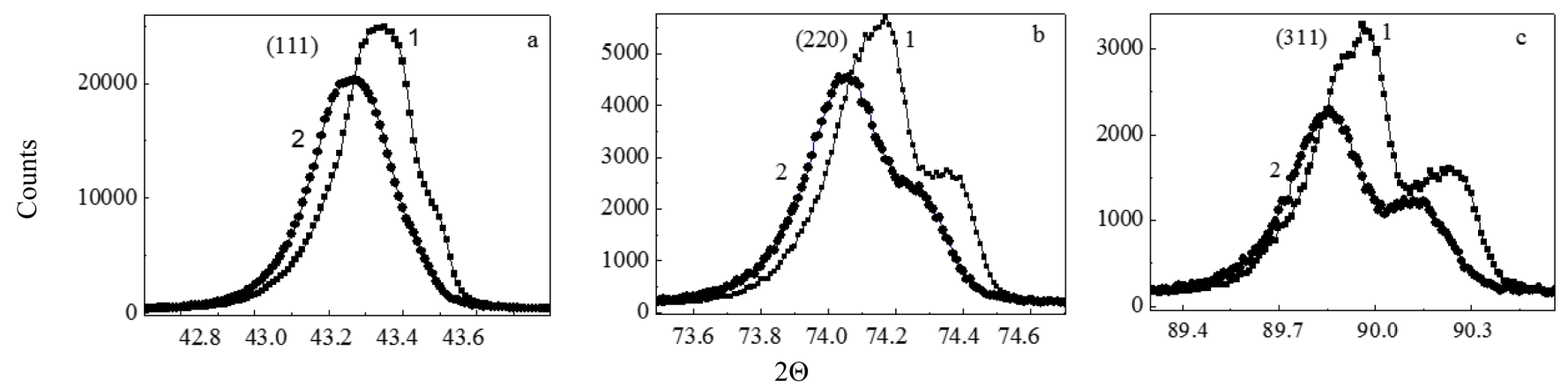

Figure 4. Peaks (111) (a); (220) (b); (311) (c) in XRD patterns of cBN:Nd micropowder with the Nd different content in cBN: 1 - < 0.01 at.\% (1), 2 - 0.05 at.\% (10).

smallest content (less than 0.01 at.\%). This effect is less expressive for $\mathrm{cBN}$ with the RE largest content, testifying to the noninstrumental nature of the asymmetry. It is known, that Guassians, Lorenzians and Voigtians are symmetric.

As seen in Figures 3(b) and Figures 3(c) and Figure 4(b) and Figures 4(c), the asymmetric peaks (220) and (311) are finely structured. This allows to suggest that XRD patterns of $\mathrm{cBN}$ :RE contain the extra-peaks which are split from the parent peaks of $\mathrm{cBN}$. When the RE concentrations in $\mathrm{cBN}$ increase (the peak 3 in Figure 3, the peak 2 in Figure 4), the peaks (220) and (311) change their profiles for smoothed shape and at first look loose the fine structure. However, if to decompose the peaks 3 and 2 into two Gaussians (dotted lines) or two Voigtians (not represented), the obtained doublets will not satisfy the requirements to $\mathrm{Cu}_{\mathrm{Ka}}$-doublet neither in intensities of their components nor in their widths. The same is related to peaks (111) and (331). Two Lorenzians absolutely are not acceptable in all cases. Thus, like the results on $\mathrm{cBN}$ :RE with the RE smallest concentration, the peaks of XRD patterns of the $c B N$ :RE with the large RE concentration also possess the more complicated structure than single $\mathrm{Cu}_{\mathrm{Ka}}$-doublets.

It is known that a small deviation from cubic symmetry of crystal lattice leads to its distortions of different types. The perfect lattice (primitive cubic, BCC, FCC) possess the singlet lines (peaks) in its XRD pattern at the use of synchrotron monochromatic irradiation. Under the polychromatic $\mathrm{Cu}_{\mathrm{Ka}}$-irradiation, all lines in the XRD pattern should be represented with $\mathrm{Cu}_{\mathrm{Kd}^{-}}$-doublets. The singlet lines (otherwise, $\alpha_{1^{-}}$and $\alpha_{2^{-}}$ component of $\mathrm{Cu}_{\dot{\alpha}}$-doublets) are characterized with multiplicity factor $m$ which is the number of reflections which superimpose in the same diffraction peak and cannot be resolved in a perfect crystal lattice, satisfying the rule for the multiplicity factor $m$. If cubic crystal lattice is distorted, additional peaks appear in the "subcubic" XRD pattern, which are split from basic peaks in XRD pattern of the parent cubic crystal lattice, breaking the rule for the $m$ factor. If to analyze the splitting scheme of the basic peaks, it is possible to define the type (tetragonal, trigonal and so on) of the distortions of cubic crystal lattice [9]-[11]. If the parent primitive or body centered or face centered cells of cubic crystal lattices are distorted, three different splitting schemes of basic peaks of the parent XRD patterns should be realized. In our mind, these schemes are not widely described in literature and we would refer the readers to [12] [13]. Worthy to add, that 
the mentioned splitting schemes are realized when all cells of a cubic crystal are uniformly distorted. The type of distortion of the FCC crystal lattice of $\mathrm{cBN}$ could be revealed when the number of extra-splits around each parent peak of XRD pattern of the material was known. The characteristics (relative integral intensity and angular distance from the corresponding parent peak) of the extra-splits allow to estimate the distortion quantitatively as well as to suggest a way of its creation. The relative integral intensity is directly connected with the part of the crystallographic planes from the multiplicity $\{\mathrm{hkl}\}$ which don't participate in formation of the (hkl) basic peak. The angular distance allows to evaluate e.g. the quantitative characteristics of tetragonal and trigonal distortions $\Delta a$ and $\Delta \gamma$ where $a$ is the parameter of cubic cell and $\gamma$ is the basic angle of the cell.

In this work, peaks (111), (220), (311) of XRD patterns of all micropowders cBN:RE were deconvoluted into Voigtians. The extra-splits (as the additional ones to peaks of the parent $\mathrm{cBN}$ ) were discovered and compared in their numbers and characteristics in dependence on the RE concentration in $\mathrm{CBN}$ and on sizes of the $\mathrm{RE}^{3+}$ ions. In correspondence with atomic numbers in the periodic table, the sizes of the RE three-charged ions should increase in the series $\mathrm{Er}^{3+}, \mathrm{Tb}^{3+}, \mathrm{Eu}^{3+}, \mathrm{Sm}^{3+}, \mathrm{Nd}^{3+}$. Any two lines resulted from the deconvolution were assigned with $\mathrm{Cu}_{\mathrm{Ka}}$-doublets if additionally to the features which were mentioned above, they possessed the similar contributions of Gaussians and Lorentzians. Making the suggestion about nonresolved $\mathrm{Cu}_{\mathrm{Ka}}$-doublets (broad peaks) as result of the deconvolution, we also kept in mind that a sum of two Voigtians is a Voigtian as well.

Being in shortage of the paper space, we represent the characteristic XRD patterns of $\mathrm{cBN}$ :RE with different doping levels. Figure 5 particularly shows the deconvoluted peaks (111), (220) and (311) in XRD patterns of cBN:Er (5) and cBN:Nd (1) (less than 0.01 at. \% RE in $\mathrm{cBN}$ ). Figure 6 particularly shows the peaks in XRD patterns of $\mathrm{cBN}: \mathrm{Tb}(12)$ and $\mathrm{cBN}: \mathrm{Sm}(10)$ with $\sim 0.05$ at. \% RE. The deconvolution is represented for the cBN:Er (20) micropowder with the RE content above 0.1 at.\% (Figure 7). As seen from Figure 5, the cBN parent peaks (111), (220) and (311) are accompanied with extra-splits at the low $2 \Theta$-position and the high $2 \Theta$-position. When the RE concentration increased up to $\sim 0.05$ at. \% RE, the parent peaks (220) and (311) lost the neighbors on the right. The extra-splits at the high $2 \Theta$-position follow only the (111) basic peaks but lost in intensity (compare Figure 6 with Figure 5). The similarly organized XRD patterns of the $\mathrm{cBN}$ :Er are just redistributed in intensity (compare Figure 7 with Figure 6). As seen, the intense basic peaks of $\mathrm{cBN}$ are there in all $\mathrm{XRD}$ patterns of $\mathrm{cBN}$ :RE confirming the presence of the $\mathrm{cBN}$ parent cells. It should be reminded, that the extra-splits at the low $2 \Theta$ and the high $2 \Theta$ arise from the planes within multiplicity $\{\mathrm{hkl}\}$, correspondingly, with the enlarged and the diminished interplanar distances, which appear in crystal lattice of $\mathrm{cBN}$ due to the RE ions' incorporation. In case, the enlarged interplanar distances $d_{h k l}$ in the $\mathrm{cBN}$ crystal lattice, appear due to large sizes of doping impurities. Possibly, that the diminished interplanar distances in the $\mathrm{cBN}$ crystal lattice can appear due to vacancies' creation which have to play the role of the mechanical strains' relaxants as well as compensators of the $\mathrm{RE}^{3+}$ ions' electrical charge. 
cBN:Er (5), cBN:Nd (1) (111)

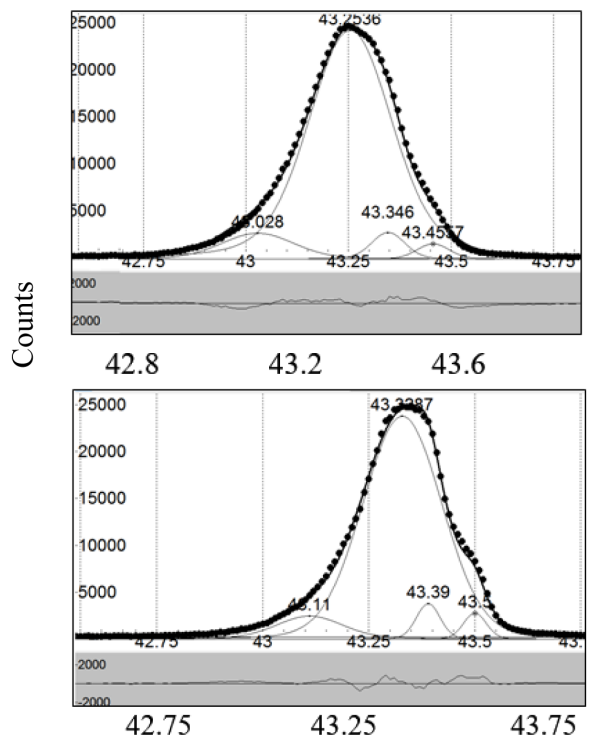

cBN:Er (5), cBN:Nd (1) (220)

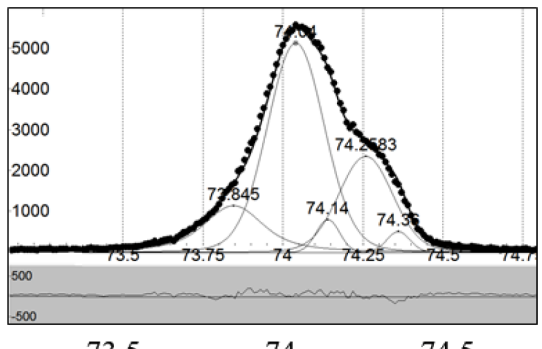

73.5

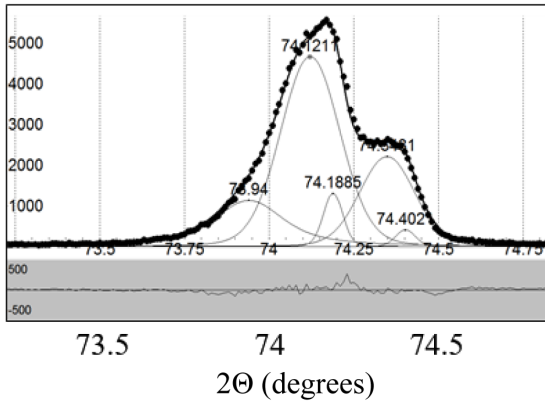

cBN:Er (5), cBN:Nd (1) (311)

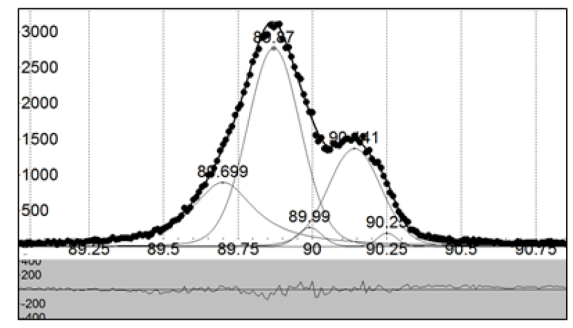

$\begin{array}{lll}89.25 & 89.75 & 90.25\end{array}$

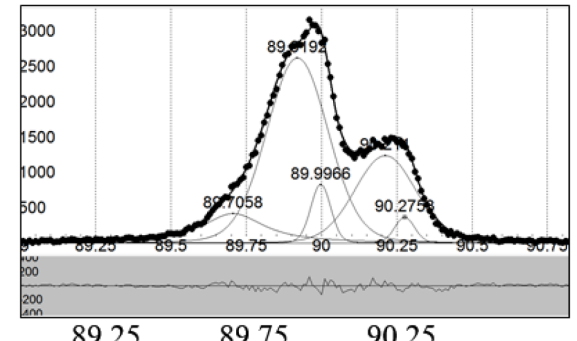

Figure 5. Peaks (111), (220) and (311) in XRD patterns of cBN:Er (5), cBN:Eu (2) and cBN:Nd (1) micropowders with the RE content less than 0.01 at.\%, deconvoluted into Voigtians which are the resolved and nonresolved $\mathrm{Cu}_{\mathrm{K} \dot{\alpha}}$-doublets.
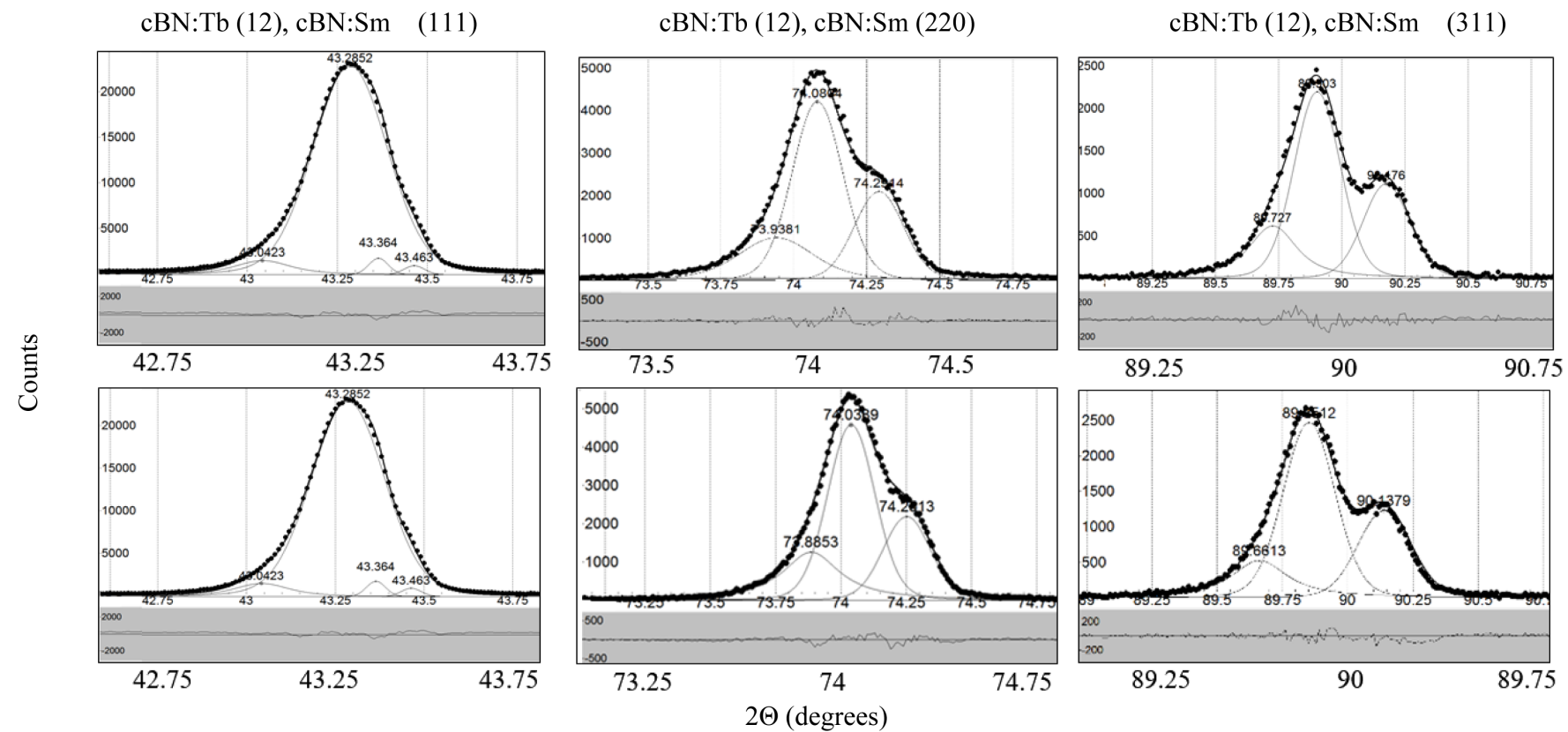

Figure 6. Peaks (111), (220) and (311) in XRD patterns of cBN:Eu (10), cBN:Tb (12) and cBN:Nd (10) micropowders with the RE content of $\sim 0.05$ at.\%, deconvoluted into Voigtians which are the resolved and nonresolved $\mathrm{Cu}_{\mathrm{Ka}}$-doublets.

It seems, that we have managed to reveal some regularities and tendencies in behavior of XRD patterns of $\mathrm{cBN}$ which is doped with impurities of large sizes. It means that we can foresee a state of the $\mathrm{cBN}$ crystal lattice and realize the doping control of $\mathrm{cBN}$ within the investigated concentrations and errors of the deconvolutions. 

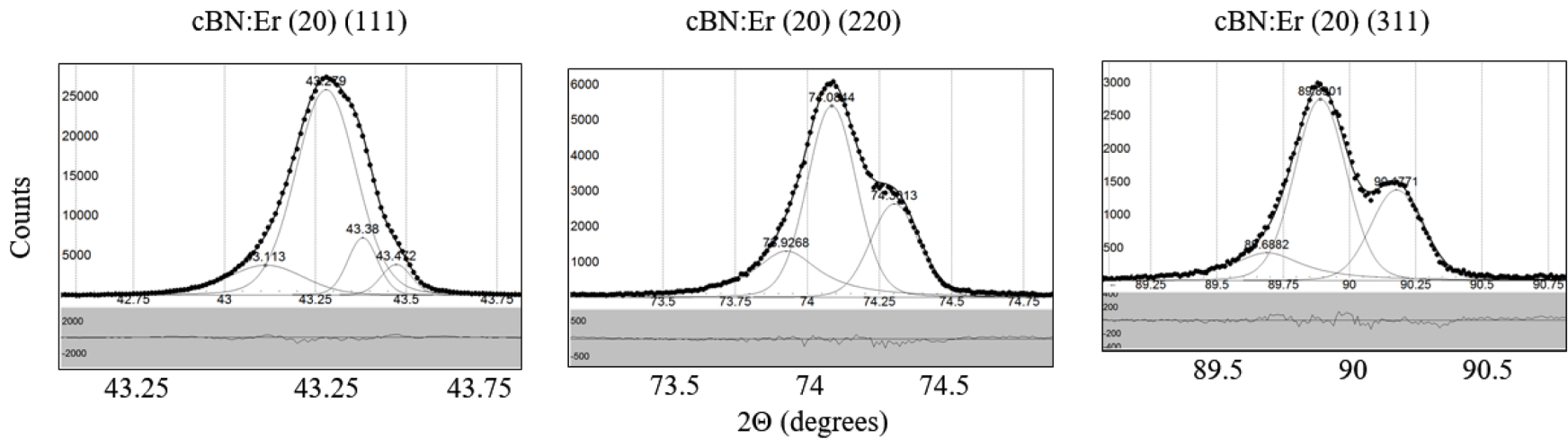

Figure 7. Peaks (111), (220) and (311) in XRD patterns of cBN:Er (20) and cBN:Tb (17) micropowders with content of Er and Tb in cBN above 0.1 at.\% are deconvoluted into Voigtians, which represent the resolved and nonresolved CuKá-doublets.

As the evaluation criteria for the latter, we selected the main characteristics of the extra-splits in XRD patterns of cBN:RE: their fixed number around each parent peak of $\mathrm{CBN}$, relative integral intensity and angular distance from the corresponding parent peak.

The number of extra-splits is clearly seen from Figures 5-7. The remaining characteristics can be analyzed from Figures 8(a)-(b') where they are mediately represented in dependence on the $\mathrm{RE}^{3+}$ ions' sizes and their concentrations in $\mathrm{cBN}$. The number of narrow extra-splits around the parent peaks (111), (220) and (311) in XRD pattern in Figure 5 cannot satisfy any splitting schemes [12] [13]. Thus, they only prove the nonuniformly distorted state of the $\mathrm{cBN}$ :RE crystal lattice with the small RE concentration. This state changes simultaneously with the RE concentration growth, but it doesn't reach the uniformity within the $\mathrm{RE}$ concentrations in $\mathrm{CBN}$ which were used here. Formally, we got that the splitting schemes could not be applied neither to the small concentrations nor to any concentrations of the $\mathrm{RE}$ in $\mathrm{CBN}$ which are investigated in the present work. Thus, the crystal lattice of $\mathrm{cBN}$ :RE represents the disordered solid solution which is nonuniformly distorted. To draw conclusions, we accept that the extra-splits are resulted from the RE ions' invasion of the $\{\mathrm{hkl}\}$ planes.

The dependences in Figure 8 let us reveal some suggestions about a possible mechanism of redistribution of the $\mathrm{RE}^{3+}$ ions inside the $\mathrm{cBN}$ crystal lattice. In $\mathrm{cBN}$ :RE with the equal concentration of different REs, the larger is the RE ion's size, the more number of (111) planes are occupied or "attacked" by the ions (Figure 8(a)); the planes (220) can be occupied by all ions except the largest ones. With the growth of the RE ion's size the planes (311) can be well occupied by the smallest ions $\left(\mathrm{Er}^{3+}\right.$ and $\left.\mathrm{Tb}^{3+}\right)$, and less with the $\mathrm{Sm}^{3+}$ ions and much less by the $\mathrm{Nd}^{3+}$ largest ions. From Figure 8(a) and Figure 8(b) follows, the more number of planes $\{\mathrm{hkl}\}$ are affected by the ions, the smaller the interplanar distances between the planes which give rise to the extra-splits around the $\mathrm{cBN}$ parent peaks in XRD patterns of $\mathrm{cBN}$ :RE. The $\mathrm{RE}^{3+}$ ions of one sort preferably try to occupy the planes $\{220\}$ and $\{311\}$ from all available planes $\{\mathrm{hkl}\}$ in crystal lattice of $\mathrm{cBN}$, except the $\mathrm{Nd}^{3+}$ ions which go into $\left.\{220)\right\}$ panes. 

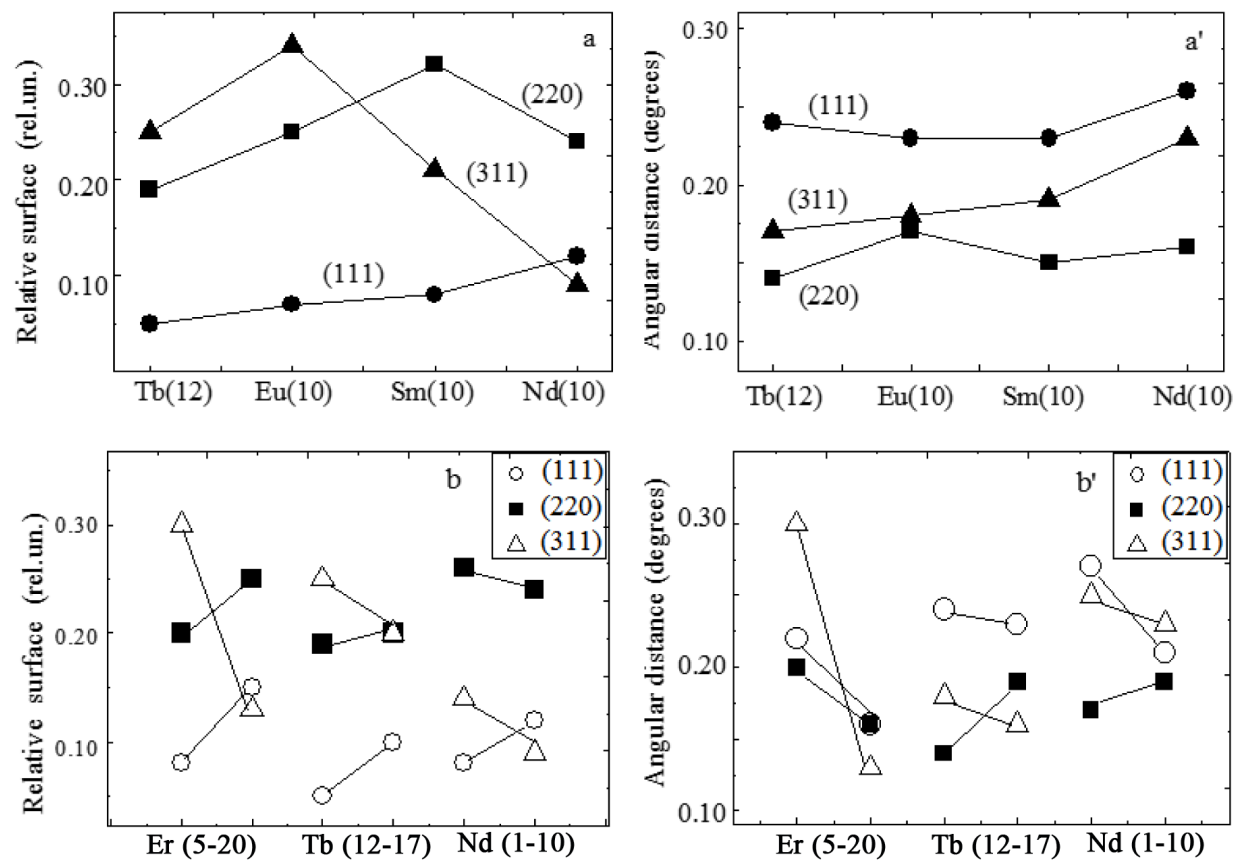

Figure 8. Dependences of the relative surface of the low $2 \Theta$ extra-split and of the angular distance between maximum of the extra-split and the corresponding basic peak (shown near the plots) in XRD patterns of cBN:RE micropowders with the similar RE concentration, on the $\mathrm{RE}^{3+}$ radius in the series $\mathrm{Er}^{3+}, \mathrm{Eu}^{3+}, \mathrm{Sm}^{3+}, \mathrm{Nd}^{3+}\left(\mathrm{a}, \mathrm{a}^{\prime}\right)$; and on the concentration of the same $\mathrm{RE}$ ions in $\mathrm{cBN}(\mathrm{b}, \mathrm{b}$ '). Concentrations of the RE compound within the total mass of the precursors of the $\mathrm{cBN}$ :RE micropowders, are shown in parentheses.

As seen from Figures $\mathbf{8}\left(\mathrm{a}^{\prime}\right)$ and Figure $\mathbf{8}\left(\mathrm{b}^{\mathbf{}}\right)$, when the concentration of the RE in the $c B N: R E$ increases, the larger number of planes $\{111\}$ and the smaller number of planes $\{311\}$ can be affected by all RE ions and the larger number of planes $\{220\}$ will be affected by all except the $\mathrm{Nd}^{3+}$ largest ions. When the concentration of the $\mathrm{RE}$ in the $\mathrm{CBN}: \mathrm{RE}$ increases, the larger number of the planes $\{111\}$ and the smaller number of planes $\{311\}$ with diminishing distances between the planes can be created in $\mathrm{cBN}$, which give rise to the extra-splits formation. Simultaneously, the number of the planes $\{220\}$ and the interplanar distances between, which give rise to the extra-splits, can vary in dependence on the RE concentration in $\mathrm{CBN}$ and on the ions' size. However, at average, the different RE ions affect the larger number of planes (220) as to compare to planes (111) and (311) when concentration of the RE in cBN increases (Figure 8( $\left.\mathrm{a}^{\prime}\right)$ ).

\section{Conclusions}

The light emitting cubic boron nitride doped with rare earth elements (cBN:RE) in form of micropowders with the RE content in $\mathrm{cBN}$ from less than 0.01 up to more than 0.1 at.\%, is synthesized, tested in IR-, red and green photoluminescence and precisely investigated using XRD analysis. New data conclusively testify $\mathrm{Eu}^{3+}$ ions to occupy two sites of low symmetry in cBN crystal lattice and to create two luminescence centers (Eu1 and Eu2). The $\mathrm{Eu}^{3+}$ ions occupy the second site in cBN crystal lattice the more ex- 
pectable, the more concentration of $\mathrm{Eu}$ in $\mathrm{cBN}$. The $\mathrm{Tb}^{3+}$ ions don't change the single position in $\mathrm{cBN}$ crystal lattice with the concentration growth as well as after similar annealing which leads the $\mathrm{Eu}^{3+}$ ions to multiply their positions and the luminescence centers in $\mathrm{CBN}$ up to four. With high probability, the intrinsic atoms of boron and nitrogen in the tetrahedral symmetry's positions can be substituted by the $\mathrm{Tb}^{3+}$ ions, because these positions are most stable and energy-optimal. It is logically to expect the smallest $\mathrm{RE}^{3+}$ ions to occupy the similar positions, and the $\mathrm{RE}^{3+}$ largest ions to have two or more crystallographic positions of low symmetry in crystal lattice of $c B N$. The latter is well supported with our previous results on $\mathrm{cBN}: \mathrm{Nd}, \mathrm{cBN}: \mathrm{Gd}$ and $\mathrm{cBN}$ :Ce.

All cBN:RE micropowders (RE: Er, Tb, Eu, Sm, Nd) showed the $\mathrm{cBN}$ single phase content under XRD analysis. In XRD patterns of $\mathrm{CBN}$ : RE, as the whole, the angular displacements of peaks to the low $2 \Theta$ positions with the RE concentration growth, did not follow the rule for the ordered solid solutions, the Bragg angle increases, the shift is the strong. This testifies to the creation of disordered solid solutions of the RE on cBN basis.

As established, all peaks in XRD patterns of $\mathrm{cBN}$ : $\mathrm{RE}$ were variously asymmetric with the asymmetry of noninstrumental nature. In consideration of the latter and the peaks' shape analysis, the narrow extra-splits around the $\mathrm{cBN}$ parent peaks were revealed and analyzed in dependence on the $\mathrm{RE}^{3+}$ ions' size and the $\mathrm{RE}$ concentrations in $\mathrm{cBN}$. As shown, the extra-splits' formation is subject to the regularities. On their basis, some suggestions are made about a possible mechanism of redistribution of the $\mathrm{RE}^{3+}$ ions inside the cBN crystal lattice.

The extra-splits' appearance in XRD patterns of the $\mathrm{cBN}$ :RE micropowders, and their regular transformations simultaneously with the cBN parent peaks' angular displacements, allow to assume that the RE large ions as create disordered solid solutions on the $\mathrm{cBN}$ basis, as bring some cells of the $\mathrm{cBN}$ parent crystal lattice to distorted states and their changes. Still, these states stay out of the classical uniform distortions of cubic crystal lattices (tetragonal, trigonal e.g). From characteristics of the extra-splits, the cBN crystal lattice is not seriously deteriorated in the investigated span of the RE concentrations. We expect new data to define a possible limit for concentrations of large impurities in $\mathrm{cBN}$ which start to break the $\mathrm{cBN}$ crystallographic identity.

\section{Acknowledgements}

E. Sh. thanks J. W Steeds (PL) and V. G. Luhin (XRD) for the measurements' providing. Without very precise PL and XRD records the work would be impossible.

\section{References}

[1] Abtew, T.A., Gao, W.W., Gao, X., Sun, Y.Y., Zhang, S.B. and Zhang, P.H. (2014) Theory of Oxygen-Boron Vacancy Defect in Cubic Boron Nitride: A Diamond NV-Isoelectronic Center. Phys. Rev. Let., 113, 134401.

[2] Gaiduk, M.I., Zolin, V.F. and Gaigerova, L.S. (1974) The Luminescence Spectra of Europium. Nauka, Moscow.

[3] Shishonok, E.M., Leonchik, S.V., Braud, A., Steeds, J.W., Abdullaev, O.R., Yakunin, A.S. 
and Zhigunov, D.M. (2010) Photolu-Minescence of Micropowders of Europium-Doped Cubic Boron Nitride. J. Opt. Technology, 77, 788-795.

[4] Shishonok, E.M. (1990) Study of the Defect-Formation Processes in Cubic Boron Nitride Using Optical Methods. Dissertation for Candidate of Physicomathematical Sciences, Minsk.

[5] Shishonok, E.M. (2009) Cubic Boron Nitride: Raman and Luminescence Investigations, Prospects for Use in Opto- and Microelectronics. Publ. Center of Belarussian State University, Minsk.

[6] Shishonok, E.M., Leonchik, S.V., Bodiou, L. and Braud, A. (2009) Photoluminescence Investigations of Cubic Boron Nitride Doped with Neodymium during High-Pressure Synthesis. Physics of the Solid State, 51, 1828-1835.

[7] Shishonok, E.M., Leonchik, S.V. and Steeds, J.W. (2008) Luminescence of Samarium-Activated cubic Boron Nitride. J. Applied Spectroscopy, 75, 89-95.

[8] Shishonok, E.M., Steeds, J.W., Pysk, A.V., Mosunov, E.O., Abdullaev, O.R., Yakunin, A.S. and Zhigunov, D.M. (2012) Structural Studies of Rare-Earth Activated Cubic Boron Nitride Micropowders. Powder Metallurgy and Metal Ceramics, 50, 754-767.

http://www.springerlink.com/openurl.asp?genre=article\&id=doi:10.1007/s11106-012-9386-5 http://dx.doi.org/10.1007/s11106-012-9386-5

[9] Darul, J. (2009) Thermal Instability of the Tetragonally Distorted Structure of Copper-Iron Materials. Z. Kristallogr. Suppl., 30, 335-340.

[10] Lindbaum, A., Heathman, S., Le Bihan, T. and Rogl, P. (2000) Pressure-Induced Orthorhombic Distortion of $\mathrm{UMn}_{2}$. J. Al loys \& Compounds, 298, 177-180.

http://dx.doi.org/10.1016/S0925-8388(99)00627-1

[11] Le Bihan, T., Idiri, M. and Heathman, S. (2003) New Investigation of Pressure-Induced Rhombohedral Distortion of Uranium Nitride. J. Alloys \& Compounds, 358, 120-125. http://dx.doi.org/10.1016/S0925-8388(03)00078-1

[12] Abakumov, A.M. www.emat.ua.ac.be/xel2006/course\%20material/X-2006CD/Abakumov/LectureAbakumov.doc

[13] Weaver, M.L. http://bama.ua.edu/ mweaver/courses/MTE583/MTE\%20583_Class_17.pdf

Submit or recommend next manuscript to SCIRP and we will provide best service for you:

Accepting pre-submission inquiries through Email, Facebook, LinkedIn, Twitter, etc. A wide selection of journals (inclusive of 9 subjects, more than 200 journals)

Providing 24-hour high-quality service

User-friendly online submission system

Fair and swift peer-review system

Efficient typesetting and proofreading procedure

Display of the result of downloads and visits, as well as the number of cited articles

Maximum dissemination of your research work

Submit your manuscript at: http://papersubmission.scirp.org/

Or contact wjet@scirp.org 